\title{
Aortic valve repair in adult congenital heart disease
}

\author{
Evaldas Girdauskas ${ }^{1,2}$, Johannes Petersen ${ }^{1,2}$, Jörg Sachweh ${ }^{3}$, Rainer Kozlik-Feldmann ${ }^{3}$, Christoph \\ Sinning ${ }^{2,4}$, Carsten Rickers ${ }^{3}$, Yskert von Kodolitsch ${ }^{4}$, Hermann Reichenspurner $^{1,2}$ \\ ${ }^{1}$ Department of Cardiac and Cardiovascular Surgery, University Heart Center Hamburg, Hamburg, Germany; ${ }^{2}$ German Center of Cardiovascular \\ Research (DZHK), Partner Site Hamburg/Kiel/Lübeck, Hamburg, Germany; ${ }^{3}$ Department of Pediatric Cardiology and Cardiac Surgery, \\ ${ }^{4}$ Department of General and Interventional Cardiology, University Heart Center Hamburg, Hamburg, Germany \\ Contributions: (I) Conception and design: E Girdauskas; (II) Administrative support: J Petersen, Y von Kodolitsch; (III) Provision of study materials or \\ patients: E Girdauskas, J Petersen; (IV) Collection and assembly of data: J Petersen, C Sinning; (V) Data analysis and interpretation: E Girdauskas, J \\ Petersen, C Rickers; (VI) Manuscript writing: All authors; (VII) Final approval of manuscript: All authors. \\ Correspondence to: Evaldas Girdauskas, MD. Department of Cardiac and Cardiovascular Surgery, University Heart Center Hamburg, Martinistr. 52, \\ 20246 Hamburg, Germany. Email: e.girdauskas@uke.de.
}

\begin{abstract}
Aortic valve repair in adult congenital heart disease (ACHD) went through a major development during the last two decades to become an increasingly established treatment option in experienced heart valve repair centers. This mini-review addresses valve-sparing treatment strategies in the two most common clinical entities of patients with adult congenital aortic valve disease, namely those presenting with bicuspid (BAV) and unicuspid (UAV) aortic valve disease. Both diseases are integral components of the continuum of congenital aortic valve diseases and represent one of the most common reasons of cardiovascular morbidity in young and otherwise healthy adult patients. The review will highlight the most important advantages of aortic valve sparing procedures as compared to the conventional valve replacement strategy. New treatment aspects will be reviewed including minimally-invasive surgical approaches for aortic valve repair as well as modern protocols of enhanced perioperative recovery which will potentially improve the perioperative recovery and quality of life of the patients undergoing valve-sparing surgical procedures in the future.
\end{abstract}

Keywords: Aortic valve repair; bicuspid aortic valve (BAV); unicuspid aortic valve (UAV); heart valve reconstruction; minimally invasive surgery; enhanced perioperative recovery

Submitted Jul 30, 2018. Accepted for publication Sep 26, 2018.

doi: $10.21037 /$ cdt.2018.11.01

View this article at: http://dx.doi.org/10.21037/cdt.2018.11.01

\section{Introduction}

Adult congenital heart disease (ACHD) prevalence is increasing over time (i.e., 119-217 cases per 100,000) and a further trend towards incremental medical complexity of this heterogeneous entity is expected $(1,2)$. Although outcomes of patients undergoing surgery for CHDs have improved significantly over the last decades, longterm survival even in those with the mild forms remains lower than that of the general population $(1,3)$. This fact emphasizes the need for close clinical surveillance of ACHD patients to understand the reasons for increased mortality while most of deaths have still a cardiovascular origin (1).

There are only limited data on the total prevalence of all forms of congenital aortic valve diseases. Large nationwide CHD databases reported mostly the prevalence of congenital aortic valve stenosis/left ventricular outflow tract (LVOT) obstruction in the range of $0.27-0.42$ per 1,000 children $(1,2)$, and most of such patients undergo surgical procedures during childhood and before reaching adulthood. Therefore, these patients are generally not suitable candidates for aortic valve repair as they present in young adulthood due to patient-prosthesis mismatch (PPM) in the ACHD out-patient units. Significant PPM has been reported in nearly half of ACHD population with an aortic valve prosthesis (4) and more than half are classified as severe PPM which are associated with a significantly reduced exercise capacity (4). Complex and 
frequently multiple redo surgical procedures are required in symptomatic patients with PPM to relieve the mismatch and to permanently reduce left ventricular afterload $(5,6)$.

Most of ACHD patients who have an untreated congenital aortic valve disease and are appropriate candidates for aortic valve repair surgery are those presenting with a bicuspid (BAV) and unicuspid (UAV) aortic valve disease (7). BAV disease is the most common congenital anomaly of the human heart with an autosomal dominant inheritance pattern and reduced penetrance (8). The prevalence of $\mathrm{BAV}$ has been historically quoted in the range between $1 \%$ and $2 \%$ with an obvious male preponderance $(9,10)$. However, these estimates are predominantly derived from necropsy studies from an earlier era and therefore may be unreliable. Furthermore, transthoracic echocardiographic surveys are limited by the fact that the sensitivity of transthoracic echocardiography to identify BAV is dependent on the specific expertise of investigator and has been reported in the range of 50-60\% $(11,12)$. Moreover, BAV disease may be associated with some familial and endemic clustering and reveal potential accumulation in certain geographic areas $(13,14)$. Therefore, the true BAV incidence in the general population is still unknown and may be underestimated due to the abovementioned limitations.

UAV disease is much more infrequent disease as compared to the BAV entity and is found in only $0.02 \%$ in the echocardiographic referral population and in $4-6 \%$ of surgical patients undergoing aortic valve surgery for aortic valve stenosis (15). UAV patients have several specific phenotypic characteristics which differ from the standard BAV cohorts and present usually with a mixed aortic valve lesion earlier in their lives for the first aortic valve intervention (15).

Quadricuspid aortic valve (QAV) is even more rare disorder that has been mostly described as a medical curiosity in the surgical and echocardiographic literature $(16,17)$. Surgical experience in QAV disease is limited to only several case reports and small surgical case series (18) and therefore will be included in the review for the sake of completeness.

This focused mini-review describes current treatment strategies of adult congenital aortic valve disease (i.e., BAV, UAV, QAV) with a special focus on aortic valve repair strategies. New perioperative aspects of surgery for congenital aortic valve diseases will be addressed which may be of value in the contemporary treatment strategies of the young adult population to achieve the desirable long-term results.

\section{Methods}

\section{Literature search}

Until July 2018, a systematic literature search was performed on PubMed, Embase, Ovid and Google Scholar databases by using the following terms: aortic valve repair, bicuspid aortic valve, unicuspid aortic valve, QAV, aortic stenosis or regurgitation, adult congenital aortic valve disease, valve sparing aortic root replacement and aortic valve reconstruction. The search was limited to original adult human studies, and papers selected were published in English at any date. All abstracts were reviewed to assess whether the article met the inclusion criteria of (I) aortic valve repair in (II) adult congenital aortic valve disease (i.e., $\mathrm{BAV}, \mathrm{UAV}$, or QAV). The key inclusion criterion was that any kind aortic valve repair was performed in congenital aortic valve lesion associated with an aortic regurgitation or/and stenosis. After this preselection process, a manual search of the reference lists of all eligible articles was performed. Two authors (i.e., E Girdauskas and J Petersen) assessed the methodological quality of the full-text articles prior to final inclusion in the manuscript.

\section{Why to repair aortic valve in ACHD?}

Major advantages of aortic valve preserving surgery result from the inherent limitations of artificial valve prostheses in valve replacement techniques. First, mechanical and biological aortic valve substitutes are far from perfect in adolescents and young adult patients as they introduce new concurring risks of anticoagulation-related complications and structural valve degeneration that have a major impact on the long-term survival and freedom from cardiovascular events (7). Mechanical aortic valve replacement has been shown to have an inferior long-term prognosis in young adult patients, as it is associated with a $27 \%$ risk of severe prosthesis-related complications-composite endpoint of death, prosthesis dysfunction, thromboembolic events, endocarditis, thrombosis of the mechanical prosthesis, and severe bleeding requiring hospitalization and transfusionduring the first 10 postoperative years after mechanical aortic valve replacement (19). The risk of reoperation on the mechanical aortic valve prosthesis was almost $20 \%$ during the first 10 postoperative years (19). Even modified anticoagulation regimes using modern $\mathrm{On}-\mathrm{X}$ mechanical valve prosthesis and low-dose marcumar therapy [i.e., target international normalized ratio (INR) of 1.5-2.0] in the PROACT trial revealed still a significant risk of 
prosthesis-related complications with a linearized rate of $4.4 \% /$ patient-year in a prospectively followed study cohort (20). Based on these data, one may summarize that a young ACHD patient who undergoes a mechanical aortic valve replacement will inevitably suffer at least one major prosthesis-related complication during the life-time (21). Bioprosthetic aortic valve substitute eliminates obviously the risk of anticoagulation-related bleeding events, introduces however the exponentially increasing risk of structural valve deterioration, requiring reoperation (22). There is an inverse relationship between age at surgery and the rate of degeneration of bioprostheses and at least half of the ACHD patients are expected to undergo reoperation within 8 postoperative years (22). Several studies reported an excess long-term mortality in young patients with aortic bioprostheses which was inversely proportional to the age of the patients at the time of surgery, i.e., younger patients had the highest mortality risk - and was further aggravated by the insertion of a small bioprosthesis (23). Valve-invalve transcatheter aortic valve replacement emerged as a novel and promising technique to address the structural degeneration of stented tissue prostheses, however, the experience with this procedure is very limited in patients less than 70 years old. There are some specific issues which might be of special relevance in the young patients presenting with a congenital aortic valve disease: increased risk of PPM with higher transvalvular gradients, risk of valve thrombosis due to incomplete unfolding of transcatheter valve-in-valve prosthesis in the stented bioprosthesis and the most relevant issue of the long-term durability of transcatheter valve-in-valve procedure in the young adults. Therefore, long-term durability data of TAVR prostheses in patients $<70$ years are urgently needed to answer the question of the appropriateness of transcatheter valve-invalve intervention in the setting of bioprosthetic aortic valve degeneration in the young adult patients presenting with congenital aortic valve disease.

Considering the endpoint of event-free life-expectancy after mechanical $v s$. biological aortic valve replacement, a meta-analysis showed a comparable event-free lifeexpectancy of 11 to 12 years in a 40-50 years adult patient independently from the type of implanted aortic valve prosthesis (24). In summary, aortic valve replacement should be treated as a palliative procedure in young ACHD patients given the fact that the available prosthetic valve material is insufficient and basically exposes young adult patients to the lifetime concurring risks of anticoagulationrelated complications vs. structural valve deterioration, requiring reintervention. From this point of view, aortic valve repair with the potential of salvage of native cusp tissue represents a viable alternative to improve the longterm event-free survival in ACHD patients (25).

\section{Principles of aortic valve repair in ACHD patients}

Techniques of aortic valve repair in ACHD patients must respect the basic Carpentier's principles of heart valve repair (26) and, therefore, to address (I) the dilated aortic valve annulus and (II) to restore the normal mobility and to recreate a sufficient coaptation line of aortic valve cusps.

\section{Aortic valve annulus}

Aortic valve annulus has a complex semilunar shape and extends from the deepest cusp insertion point in the LVOT to the sinotubular junction (STJ) in the commissural area (27). Therefore, from a very practical point of view the dilatation of aortic valve annulus may be subdivided into three categories: (I) dilatation at the level of deepest insertion point of aortic valve cusps in the LVOT (so-called "aorto-ventricular junction or basal ring") (Figure 1A), (II) dilatation at the STJ level (Figure 1B), and dilatation of the whole aortic root including basal ring and STJ (Figure 1C).

Depending on the form of aortic valve annular dilatation, annuloplasty is performed using a specific technique. In ACHD patients with an isolated dilation of the basal ring (Figure 1A) suture (28) or ring annuloplasty (29) is performed at the level of basal ring. Patients with STJ dilatation (Figure 1B) have mostly a concomitant ascending aortic aneurysm and STJ reduction is achieved by using an ascending aortic graft prosthesis with an appropriate diameter. In case of annular dilatation involving the whole aortic root (Figure 1C) valve sparing root surgery (i.e., reimplantation or remodeling) is performed and stabilizes both annular components at the level of basal ring as well as STJ.

\section{Cusp disease}

Cusp disease in aortic regurgitation patients is subdivided according to the functional Carpentier's classification which has been adopted to aortic valve disease by Dr. El Khoury group in Brussels (30). Based on the mobility of aortic valve cusps (i.e., normal, prolapse and restriction) three types of cusp disease can be separated which are addressed by specific surgical techniques (Figure 2). 
A
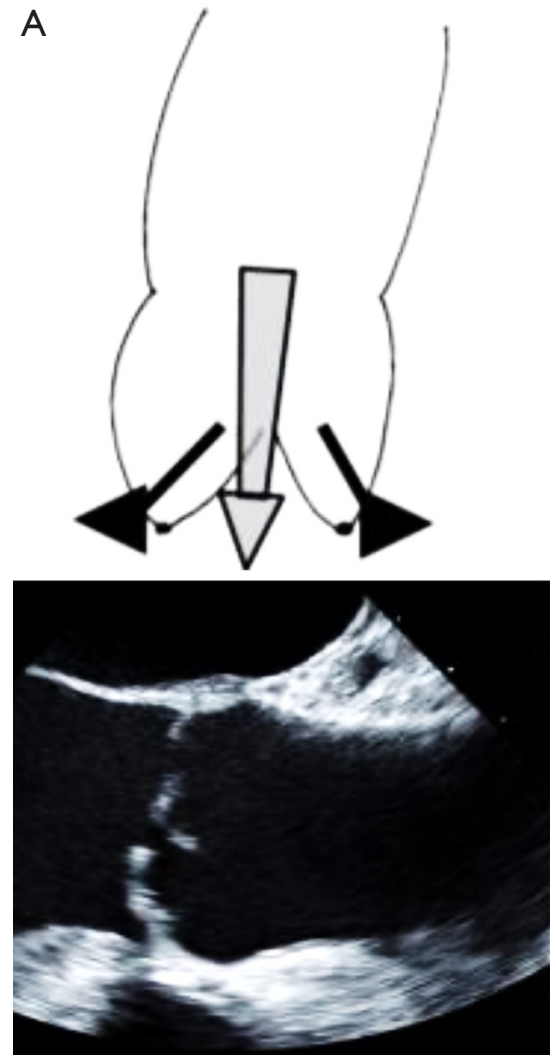

B
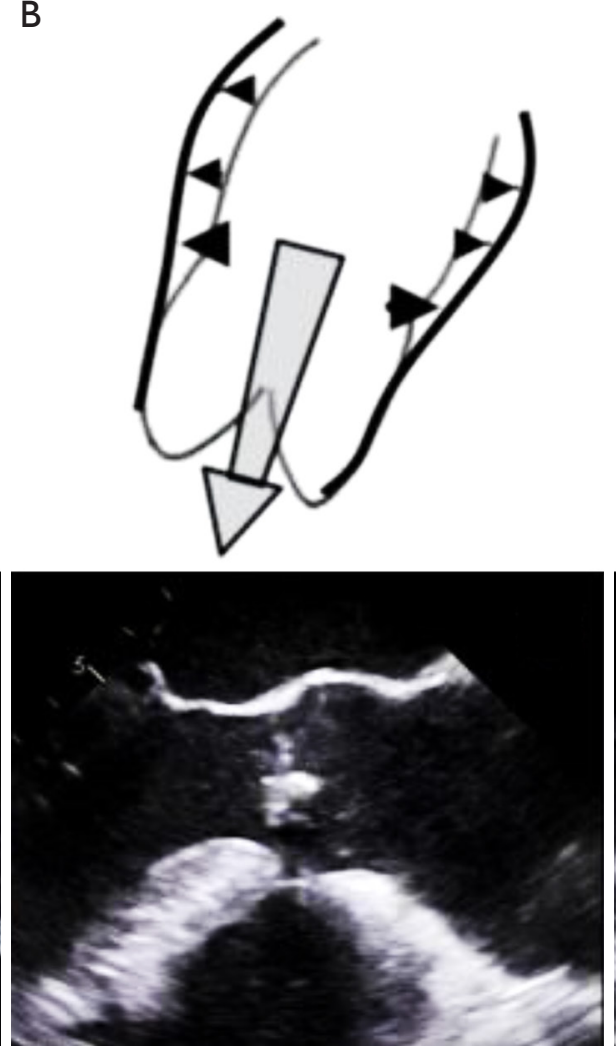

C
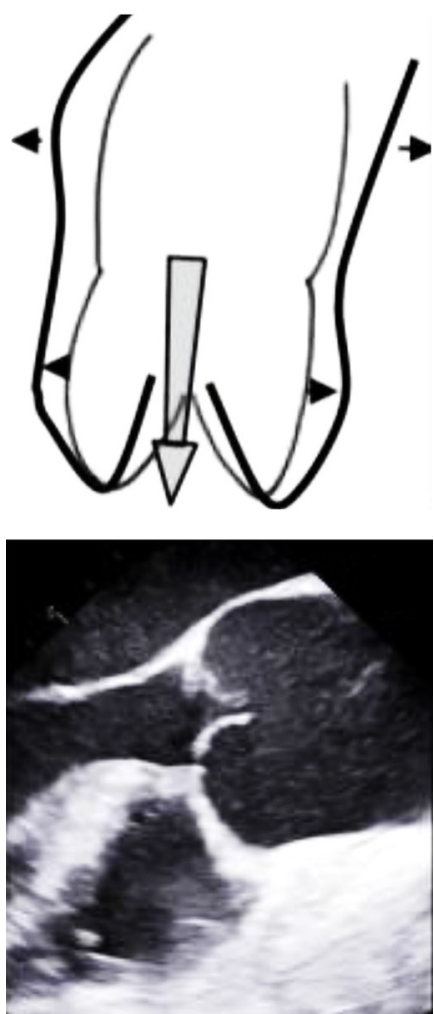

Figure 1 Forms of aortic valve annulus dilatation in ACHD patients. (A) Dilatation of the basal ring; (B) STJ dilatation; (C) dilatation of the whole aortic root. ACHD, adult congenital heart disease; STJ, sinotubular junction.

In type I cusp disease the mechanism of aortic regurgitation is annulus dilatation which is addressed as described previously (Figure $2 A$ ). In type II cusp disease the cusp prolapse is corrected by central plication sutures or a limited triangular resection (Figure 2B). Given the absolute or relative lack of functioning native cusp tissue, type III cusp disease is the most challenging cusp pathology for repair procedures. Resection of the restrictive cusp tissue and subsequent patch augmentation is often required which obviously limits the durability of repair due to structural degradation of patch material (Figure 2C).

\section{Aortic valve repair in BAV disease}

BAV is a very heterogeneous entity and distinct valvuloaortic phenotypes have been previously reported (31). Valvular morphotypes of BAV have been classified according to the Sievers classification model that integrates the number of raphes (type 0 , type 1 , and type 2), cusp fusion pattern [fusion of the right- and left-coronary cusp
(R/L), fusion of right- and non-coronary cusp (R/N)] and functional type of valve lesion (stenosis, insufficiency and mixed disease) (32). The most common form is type $1 \mathrm{BAV}$ with a single raphe between the right and left coronary cusp which is associated with a stenotic lesion (i.e., Sievers type 1, $\mathrm{R} / \mathrm{L}, \mathrm{S}$ ) and is found in more than half of all BAV patients (3). Furthermore, BAV has been shown to be associated with different types of concomitant aortopathy (33) and distinct classification models of aortic phenotypes have been developed $(34,35)$. During the ongoing development of aortic valve sparing techniques in the BAV disease, more delicate morphologic details of BAV structure came into the focus of interest, especially the angle of commissural orientation (36), the geometric and effective cusp height (37), and the pathophysiological mechanism of aortic regurgitation. Therefore, in terms of BAV treatment strategy (i.e., repair $v s$. replacement), there is an urgent need to update the previously published BAV classification models to include the parameters crucial for aortic valve repair techniques.

Despite the above-mentioned phenotypic heterogeneity 

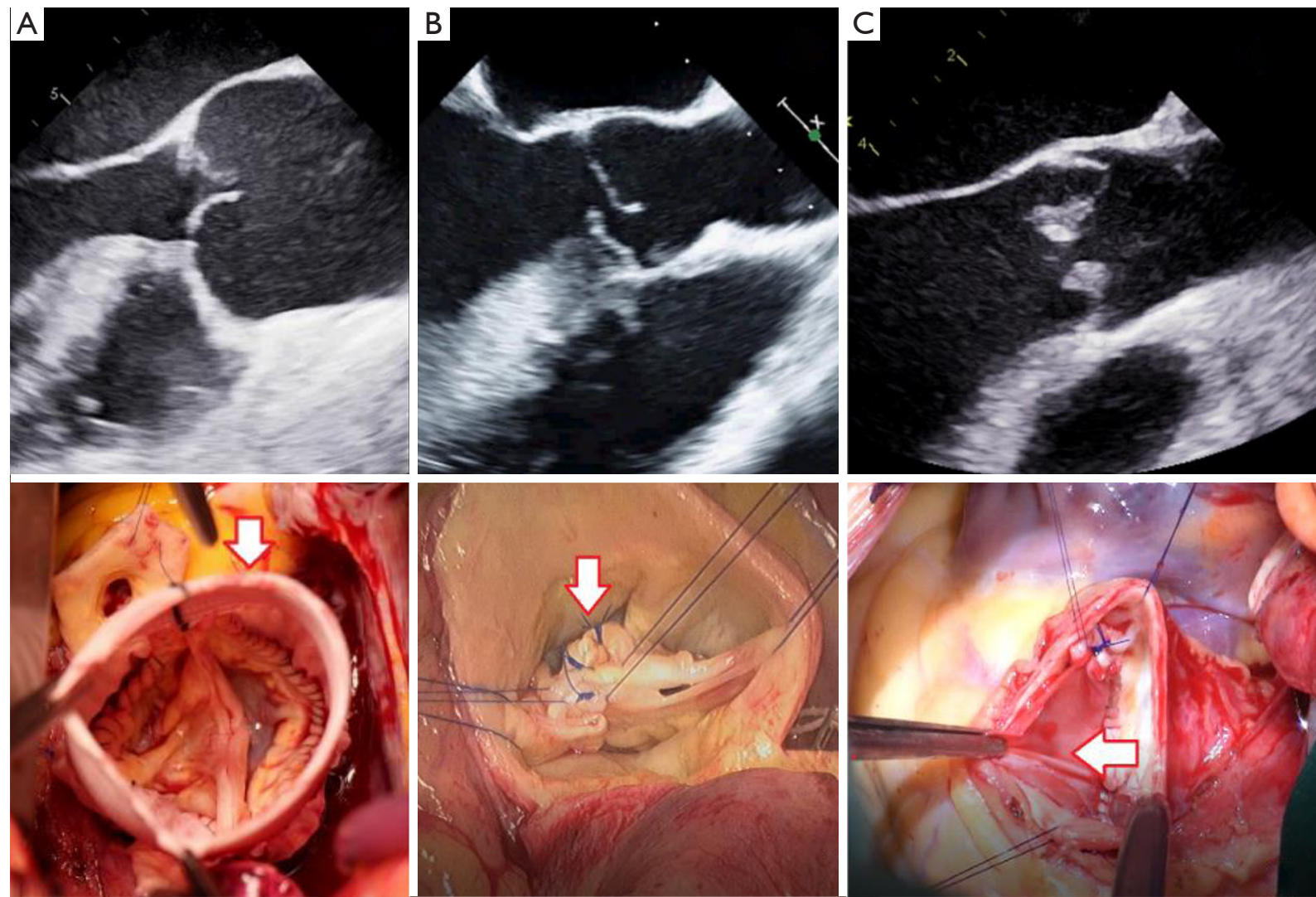

Figure 2 Types of cusp disease in ACHD patients and the corresponding surgical techniques. (A) Type I: normal cusp movement and the surgical technique of valve sparing root surgery. Arrow indicates Dacron prosthesis which is used for reimplantation procedure; (B) type II: cusp prolapse addressed by central plication of the fused cusp. Arrow indicates cusp plication in the central part of the cusp to correct the prolapse; (C) type III: restrictive cusp movement treated by cusp augmentation using pericardial patch. Arrow indicates decellularized bovine pericardial patch implantation to address cusp restriction. ACHD, adult congenital heart disease.

of BAV disease (31,32), BAV patients represent the most common morphological substrate for aortic valve interventions in young adults (38). Almost one third of BAV patients develop a hemodynamically relevant aortic valve regurgitation at the mean age of 45 years and are faced a complex decision-making process regarding the most appropriate aortic valve intervention (39). Most BAV patients with an isolated/predominant aortic regurgitation are very suitable candidates for aortic valve repair strategy which is based on two congenital-morphologic components of bicuspidality which result in geometric alteration of functional aortic root: (I) dilatation of the aorto-ventricular junction (so-called "basal ring") is a classical congenitalmorphologic feature of bicuspidality which results in increased shear stress on aortic valve cusps (Figure $3 A$ ); (II) prolapse (or restriction) of the conjoined (so-called "fused") cusp which reduces effective cusp height and causes coaptation loss of the fused cusp (Figure 3B,C). Both abovementioned morphologic substrates of $\mathrm{BAV}$ regurgitation are amenable for valve sparing techniques.

Additionally, one half of all BAV regurgitation patients have a concomitant proximal aortopathy which enlarges significantly the diameter of STJ and thereby aggravates further geometric alterations of the functional aortic root.

The main idea of aortic valve repair strategy in BAV, and altogether in adult congenital aortic valve disease, is that the recreation of the optimal aortic root geometryi.e., reduction of basal ring diameter to less than $25 \mathrm{~mm}$ and restoration of effective cusp height (coaptation length) above 8 -mm-would result in the competence of aortic valve and the long-term stability of bicuspid architecture (40). In other words, the expectation is that recreation of the symmetric, best possible geometric configuration of the regurgitant BAVs will significantly reduce the degeneration rate of 

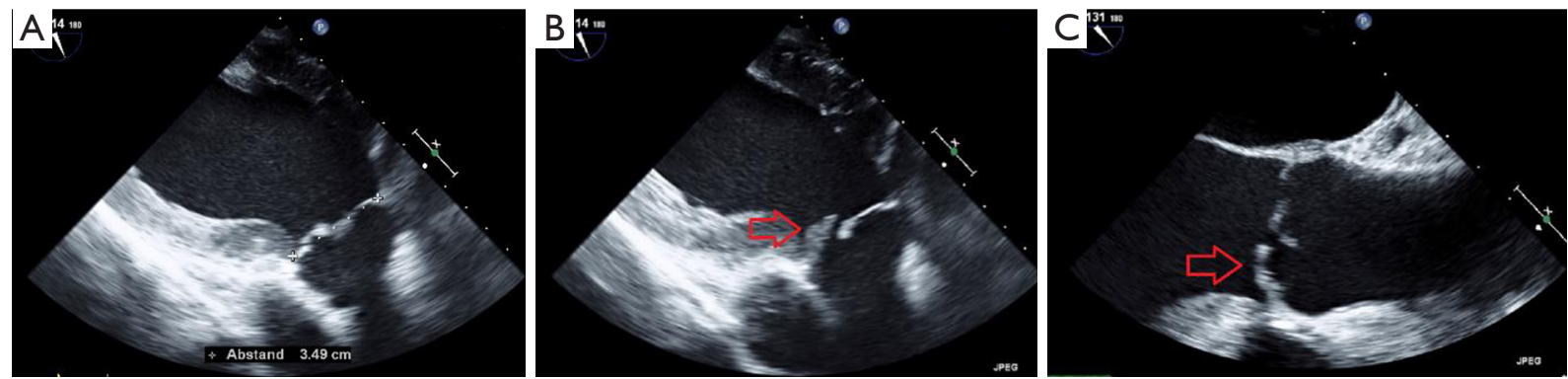

Figure 3 The most common congenital-morphologic substrate of BAV regurgitation. Arrows indicate prolapse of the fused cusp. BAV, bicuspid aortic valve.
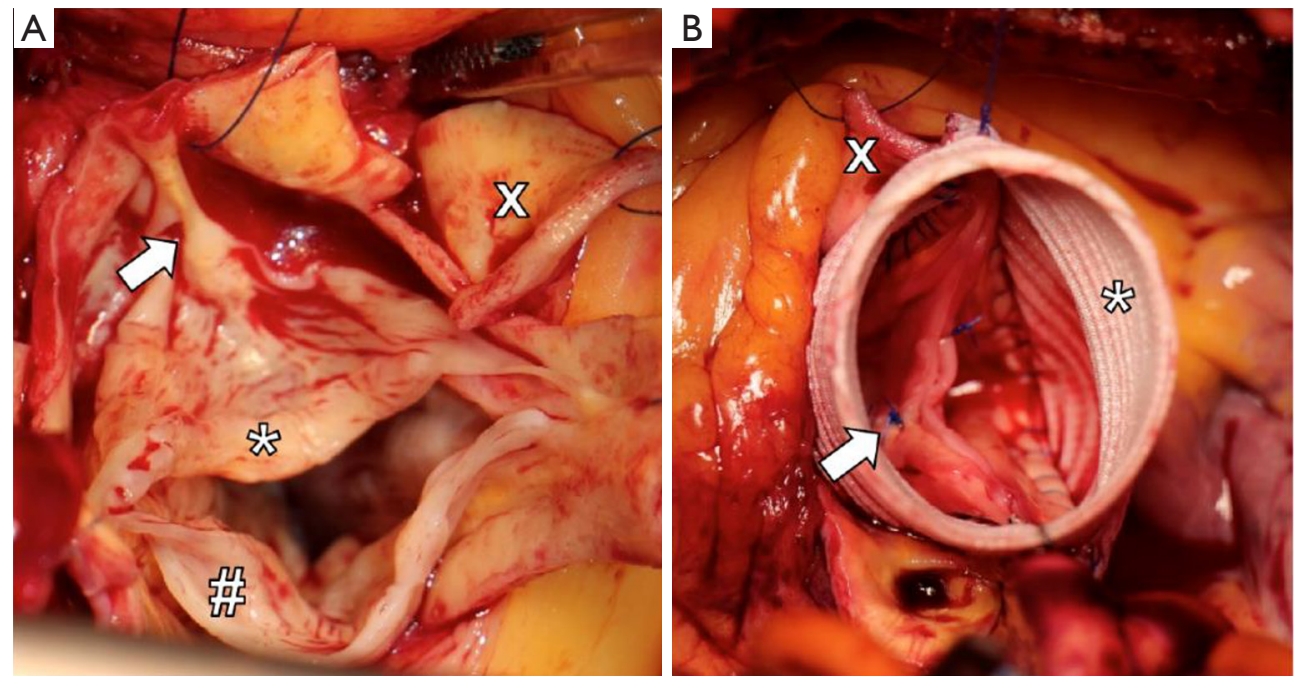

Figure 4 Recreation of the geometric configuration of the functional bicuspid aortic root. (A) Asymmetric Sievers type I R/L BAV with dilatation of basal ring to $34 \mathrm{~mm}$, prolapse of the fused cusp $\left(^{*}\right)$, normal non-coronary cusp (\#) and aneurysm of the aortic root which has been excised. Arrow indicates raphe between right and left coronary cusp; (B) valve sparing root replacement by remodeling technique ${ }^{*}$ ) with an additional reduction of basal ring diameter to $25 \mathrm{~mm}$, prolapse correction of both cusps (arrow) and recreation of the symmetric configuration of bicuspid valve. Cross marks indicate the bottom of right coronary ostium.

bicuspid cusp tissue and improve long-term stability of such valves (Figure 4). Retrospective monocentric data support strongly this argumentation (40-42), however, has still to be validated by ongoing prospective multicenter trials.

\section{Aortic valve repair in UAV disease}

$\mathrm{UAV}$ is defined as aortic valve with one fully developed commissure and two raphes at the level of two non-functional commissures (15) (Figure 5).

The published evidence in the literature suppose that UAV disease represent an extreme in the spectrum of adult congenital aortic valve disorders, as UAV patients generally present with a more accelerated and symptomatic aortic valve degeneration and stenosis at younger age as compared to the BAV patients (43). Most reports of UAV patients describe young individuals with advanced valvular disease that develops early and progresses at a faster rate than typical BAV. The challenge of aortic valve repair techniques in UAV patients is the absolute lack of functional aortic cusp tissue which is frequently very dysplastic and severely calcified in the areas of both rudimentary commissures (i.e., type III cusp disease). This tissue lack results in a central coaptation defect and a wide central jet with several eccentric components due to restrictive cusp movement. From a technical standpoint UAV can be transformed into 

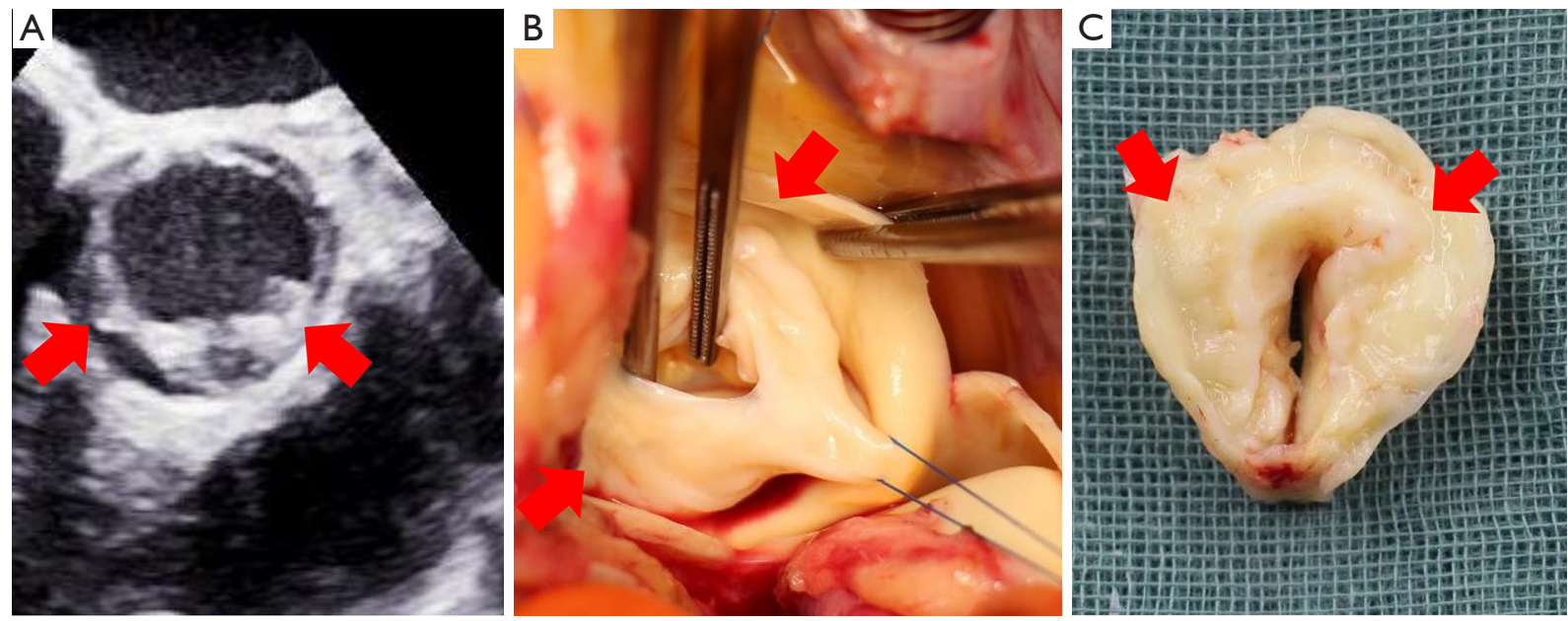

Figure 5 Echocardiographic and intraoperative appearance of the unicuspid aortic valve. Red arrows indicate: (A) two fused commissures and a typical posterior opening towards the left atrium of UAV in a transesophageal echocardiographic image; (B,C) and the intraoperative appearance of unicuspid aortic valve before and after excision during the surgery.

a symmetric BAV morphology using an augmentation patch (i.e., autologous pericardium or decellularized bovine pericardium) to reconstruct the second functional neo-commissure, as published by Dr. Schäfers group in Homburg (44) (see Figure 2C). However, patch repair has been shown to be a risk factor that reduces repair durability and is driven by the structural deterioration of the patch material. Nonetheless, in growing patients with small LVOT and aortic annulus diameter UAV repair even with a patch represents a viable treatment option because it allows the native annulus to grow normally and thereby prevents PPM occurrence. This strategy also allows the eventual Ross operation to be delayed to an adulthood where the pulmonary homograft is better tolerated.

\section{QAV and the preliminary experience with repair techniques}

QAV is a very rare cardiac condition with a reported prevalence of $0.008 \%$ to $0.46 \%$ depending on the study population analyzed. The functional status of QAV is predominantly regurgitant (45), however, the experience with aortic valve sparing techniques in the setting of regurgitant QAV is still limited to few published case reports and no systematic reconstruction approach has been yet developed. Depending on the specific morphologic characteristics of QAV a tricuspidalization $v s$. bicuspidalization repair strategy has been proposed for QAV repair with a varying success (46-48).

\section{Minimally invasive approach in aortic valve repair of ACHD patients}

The ongoing development of surgical technologies, routine implementation of the intraoperative transesophageal echocardiography monitoring and the growing experience with video-assisted surgery allowed the standard use of limited surgical approaches in aortic valve surgery. Standard use of the partial upper sternotomy in the 3 or 4 . intercostal space reduce significantly surgical trauma and provides an excellent access to the aortic valve and the proximal aorta and thereby enables the whole spectrum of surgical procedures on the aortic valve and the aortic root/ascending aorta (Figure 6). In addition to the superior cosmetic result and the reduction of sternal wound complications after the minimallyinvasive surgery, recent meta-analysis of randomized trials documented reduced postoperative blood loss and lower need for blood transfusion in the partial sternotomy subgroup $v s$. conventional sternal access (49). Even if the hard study endpoints-i.e., hospital mortality, stroke, cardiovascular complications, renal failure as well as ICU and hospital stay-were not significantly different between the partial $v s$. full sternotomy subgroups in the published prospective randomized trials $(50,51)$, the standard use of minimallyinvasive approach did not negatively affect the safety profile of aortic valve surgery. From our personal point of view, even the evidence of non-inferiority of minimally-invasive approaches in aortic valve repair surgery in ACHD patients legitimates the routine use of limited surgical access in the 

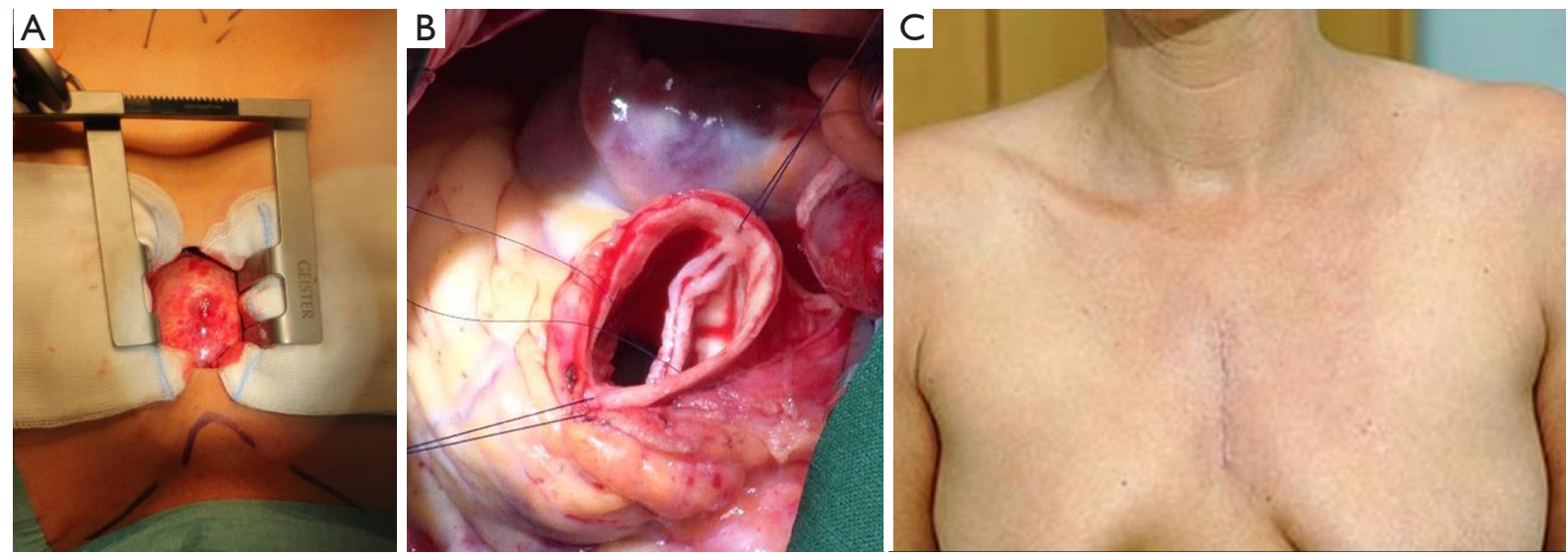

Figure 6 Complex aortic valve repair using a partial upper sternotomy approach.

daily practice of aortic valve/aortic root surgery and results in better patients' acceptance.

\section{Summary}

Surgical treatment of adult congenital aortic valve diseases, and especially aortic valve sparing techniques underwent major development during the last decades and improved significantly the outcomes and quality of life of such patients. Specific surgical strategies have been developed to address BAV and UAV disease and to avoid the life-long risk of prosthesis-associated complications of mechanical and biological aortic valve substitutes in young adult population. This mini-review discussed new treatment aspects of aortic valve lesions in ACHD patients and presented current scientific evidence for aortic valve sparing techniques and shed some light on the future developments in this innovative field of cardiovascular medicine.

\section{Acknowledgements}

None.

\section{Footnote}

Conflicts of Interest: The authors have no conflicts of interest to declare.

\section{References}

1. Wu MH, Lu CW, Chen HC, et al. Adult Congenital Heart Disease in a Nationwide Population 2000-2014:
Epidemiological Trends, Arrhythmia, and Standardized Mortality Ratio. J Am Heart Assoc 2018;7.

2. Marelli AJ, Mackie AS, Ionescu-Ittu R, et al.

Congenital heart disease in the general population: changing prevalence and age distribution. Circulation 2007;115:163-72.

3. Spector LG, Menk JS, Knight JH, et al. Trends in LongTerm Mortality After Congenital Heart Surgery. J Am Coll Cardiol 2018;71:2434-46.

4. van Slooten YJ, van Melle JP, Freling HG, et al. Aortic valve prosthesis-patient mismatch and exercise capacity in adult patients with congenital heart disease. Heart 2016;102:107-13.

5. Brown JW, Ruzmetov M, Fiore AC, et al. Long-term results of apical aortic conduits in children with complex left ventricular outflow tract obstruction. Ann Thorac Surg 2005;80:2301-8.

6. Bilal MS, Yerebakan C, Karaci AR, et al. Ross-Konno procedure for the surgical treatment of prosthesis-patient mismatch after prosthetic aortic valve replacement. J Card Surg 2013;28:666-9.

7. d'Udekem Y. Aortic valve surgery in children. Heart 2011;97:1182-9.

8. Cripe L, Andelfinger G, Martin LJ, et al. Bicuspid aortic valve is heritable. J Am Coll Cardiol 2004;44:138-43.

9. Roberts WC. The congenitally bicuspid aortic valve. A study of 85 autopsy cases. Am J Cardiol 1970;26:72-83.

10. Pomerance A. Pathogenesis of aortic stenosis and its relation to age. Br Heart J 1972;34:569-74.

11. Hillebrand M, Koschyk D, Ter Hark P, et al. Diagnostic accuracy study of routine echocardiography for bicuspid aortic valve: a retrospective study and meta-analysis. 
Cardiovasc Diagn Ther 2017;7:367-79.

12. Alegret JM, Palazón O, Duran I, et al. Aortic valve morphology definition with transthoracic combined with transesophageal echocardiography in a population with high prevalence of bicuspid aortic valve. Int J Cardiovasc Imaging 2005;21:213-7.

13. Huntington K, Hunter AG, Chan KL. A prospective study to assess the frequency of familial clustering of congenital bicuspid aortic valve. J Am Coll Cardiol 1997;30:1809-12.

14. Chandra S, Lang RM, Nicolarsen J, et al. Bicuspid aortic valve: inter-racial difference in frequency and aortic dimensions. JACC Cardiovasc Imaging 2012;5:981-9.

15. Mookadam F, Thota VR, Garcia-Lopez AM, et al. Unicuspid aortic valve in adults: a systemic review. J Heart Valve Dis 2010;19:79-85

16. Plaunova A, Gulkarov I, Tortolani AJ, et al. Surgery for a quadricuspid aortic valve: case report and comprehensive review of the literature. J Heart Valve Dis 2015;24:260-2.

17. Das A, Singh U, Rajashekar P. Quadricuspid aortic valve: A rare intraoperative diagnosis by transesophageal echocardiography. Ann Card Anaesth 2018;21:95-6.

18. Hayakawa M, Asai T, Kinoshita T, et al. Quadricuspid aortic valve: a report on a 10-year case series and literature review. Ann Thorac Cardiovasc Surg 2014;20 Suppl:941-4.

19. Bouhout I, Stevens LM, Mazine A, et al. Long-term outcomes after elective isolated mechanical aortic valve replacement in young adults. J Thorac Cardiovasc Surg 2014;148:1341-6.e1.

20. Puskas J, Gerdisch M, Nichols D, et al. Reduced anticoagulation after mechanical aortic valve replacement: interim results from the prospective randomized on-X valve anticoagulation clinical trial randomized Food and Drug Administration investigational device exemption trial. J Thorac Cardiovasc Surg 2014;147:1202-10.

21. Mazine A, Ouzounian M. Aortic valve replacement in young and middle-aged adults: looking beyond the tree that hides the forest. Ann Transl Med 2017;5:92.

22. Alsoufi B, Manlhiot C, McCrindle BW, et al. Aortic and mitral valve replacement in children: is there any role for biologic and bioprosthetic substitutes? Eur J Cardiothorac Surg 2009;36:84-90; discussion 90.

23. Mihaljevic T, Nowicki ER, Rajeswaran J, et al. Survival after valve replacement for aortic stenosis: implications for decision making. J Thorac Cardiovasc Surg 2008;135:1270-8; discussion 1278-9.

24. van Geldorp MW, Eric Jamieson WR, Kappetein AP, et al. Patient outcome after aortic valve replacement with a mechanical or biological prosthesis: weighing lifetime anticoagulant-related event risk against reoperation risk. J Thorac Cardiovasc Surg 2009;137:881-6, 886e1-5.

25. Aicher D, Fries R, Rodionycheva S, et al. Aortic valve repair leads to a low incidence of valve-related complications. Eur J Cardiothorac Surg 2010;37:127-32.

26. Carpentier A. Cardiac valve surgery--the "French correction". J Thorac Cardiovasc Surg 1983;86:323-37.

27. Anderson RH. The surgical anatomy of the aortic root. Multimed Man Cardiothorac Surg 2007;2007:mmcts.2006.002527.

28. Schneider U, Hofmann C, Aicher D, et al. Suture Annuloplasty Significantly Improves the Durability of Bicuspid Aortic Valve Repair. Ann Thorac Surg 2017;103:504-10.

29. Lansac E, Di Centa I, Sleilaty G, et al. Long-term results of external aortic ring annuloplasty for aortic valve repair. Eur J Cardiothorac Surg 2016;50:350-60.

30. Boodhwani M, de Kerchove L, Glineur D, et al. Repairoriented classification of aortic insufficiency: impact on surgical techniques and clinical outcomes. J Thorac Cardiovasc Surg 2009;137:286-94.

31. Padang R, Bannon PG, Jeremy R, et al. The genetic and molecular basis of bicuspid aortic valve associated thoracic aortopathy: A link to phenotype heterogeneity. Ann Cardiothorac Surg 2013;2:83-91.

32. Sievers HH, Schmidtke C. A classification system for the bicuspid aortic valve from 304 surgical specimens. J Thorac Cardiovasc Surg 2007;133:1226-33.

33. Fernandes S, Khairy P, Graham DA, et al. Bicuspid aortic valve and associated aortic dilation in the young. Heart 2012;98:1014-9.

34. Della Corte A, Bancone C, Dialetto G, Cet al. The ascending aorta with bicuspid aortic valve: a phenotypic classification with potential prognostic significance. Eur J Cardiothorac Surg 2014;46:240-7; discussion 247.

35. Fazel SS, Mallidi HR, Lee RS, et al. The aortopathy of bicuspid aortic valve disease has distinctive patterns and usually involves the transverse aortic arch. J Thorac Cardiovasc Surg 2008;135:901-7, 907.e1-2.

36. Lansac E, de Kerchove L. Aortic valve repair techniques: state of the art. Eur J Cardiothorac Surg 2018;53:1101-7.

37. Schäfers HJ, Schmied W, Marom G, et al. Cusp height in aortic valves. J Thorac Cardiovasc Surg 2013;146:269-74.

38. Roberts WC, Ko JM. Frequency by decades of unicuspid, bicuspid, and tricuspid aortic valves in adults having isolated aortic valve replacement for aortic stenosis, with or without associated aortic regurgitation. Circulation 2005;111:920-5. 
39. Masri A, Svensson LG, Griffin BP, et al. Contemporary natural history of bicuspid aortic valve disease: a systematic review. Heart 2017;103:1323-30.

40. Aicher D, Kunihara T, Abou Issa O, et al. Valve configuration determines long-term results after repair of the bicuspid aortic valve. Circulation 2011;123:178-85.

41. Vohra HA, Whistance RN, De Kerchove L, et al. Valvepreserving surgery on the bicuspid aortic valve. Eur J Cardiothorac Surg 2013;43:888-98.

42. Svensson LG, Al Kindi AH, Vivacqua A, et al. Long-term durability of bicuspid aortic valve repair. Ann Thorac Surg 2014;97:1539-47.

43. Krepp JM, Roman MJ, Devereux RB, et al. Bicuspid and unicuspid aortic valves: Different phenotypes of the same disease? Insight from the GenTAC Registry. Congenit Heart Dis 2017;12:740-5.

44. Schäfers HJ, Aicher D, Riodionycheva S, et al. Bicuspidization of the unicuspid aortic valve: a new reconstructive approach. Ann Thorac Surg 2008;85:2012-8.

45. Yuan SM. Quadricuspid Aortic Valve: A Comprehensive Review. Braz J Cardiovasc Surg 2016;31:454-60.

Cite this article as: Girdauskas E, Petersen J, Sachweh J, Kozlik-Feldmann R, Sinning C, Rickers C, von Kodolitsch Y, Reichenspurner H. Aortic valve repair in adult congenital heart disease. Cardiovasc Diagn Ther 2018;8(6):789-798. doi: 10.21037/ cdt.2018.11.01
46. Daprati A, Generali T, Arlati F, Roberto M. Quadricuspid aortic valve plasty: is it worth it to repair as an alternative to substitution? Ann Thorac Surg 2013;95:e7-8.

47. Bouhout I, El-Hamamsy I, Raboisson MJ, et al. Bicuspization of a quadricuspid truncal valve. Multimed Man Cardiothorac Surg 2017;2017.

48. Ohira S, Doi K, Yamano T, et al. Early failure of quadricuspid aortic valve tricuspidization due to dehiscence of approximating coronary cusps. J Echocardiogr 2014;12:75-7.

49. Kirmani BH, Jones SG, Malaisrie SC, et al. Limited versus full sternotomy for aortic valve replacement. Cochrane Database Syst Rev 2017;4:CD011793.

50. Furukawa N, Kuss O, Aboud A, et al. Ministernotomy versus conventional sternotomy for aortic valve replacement: matched propensity score analysis of 808 patients. Eur J Cardiothorac Surg 2014;46:221-6

51. Shehada SE, Elhmidi Y, Mourad F, et al. Minimal access versus conventional aortic valve replacement: a metaanalysis of propensity-matched studies. Interact Cardiovasc Thorac Surg 2017;25:624-32. 